\title{
How well can the evolution of the scale factor be reconstructed by the current data?
}

\author{
Sandro D. P. Vitenti ${ }^{1}$ and Mariana Penna-Lima ${ }^{2}$ \\ ${ }^{1}$ GReCO, Institut d'Astrophysique de Paris, \\ UMR7095 CNRS, 98 bis boulevard Arago, 75014 Paris, France \\ email: dias@iap.fr \\ ${ }^{2}$ Divisão de Astrofísica, Instituto Nacional de Pesquisas Espaciais, \\ Av. dos Astronautas 1758, 12227-010, São José dos Campos, Brazil \\ email: mariana.lima@inpe.br
}

\begin{abstract}
Distance measurements are currently the most powerful tool to study the expansion history of the universe without assuming its matter content nor any theory of gravitation. In general, the reconstruction of the scale factor derivatives, such as the deceleration parameter $q(z)$, is computed using two different methods: fixing the functional form of $q(z)$, which yields potentially biased estimates, or approximating $q(z)$ by a piecewise nth-order polynomial function, whose variance is large. In this work, we address these two methods by reconstructing $q(z)$ assuming only an isotropic and homogeneous universe. For this, we approximate $q(z)$ by a piecewise cubic spline function and, then, we add to the likelihood function a penalty factor, with scatter given by $\sigma_{r e l}$. This factor allows us to vary continuously between the full $n$ knots spline, $\sigma_{\text {rel }} \rightarrow \infty$, and a single linear function, $\sigma_{\text {rel }} \rightarrow 0$. We estimate the coefficients of $q(z)$ using the Monte Carlo approach, where the realizations are generated considering $\Lambda \mathrm{CDM}$ as a fiducial model. We apply this procedure in two different cases and assuming four values of $\sigma_{\text {rel }}$ to find the best balance between variance and bias. First, we use only the Supernova Legacy Survey 3-year (SNLS3) sample and, in the second analysis, we combine the type Ia supernova (SNeIa) likelihood with those of baryonic acoustic oscillations (BAO) and Hubble function measurements. In both cases we fit simultaneously $q(z)$ and 4 nuisance parameters of the supernovae, namely, the magnitudes $\mathcal{M}_{1}$ and $\mathcal{M}_{2}$ and the light curve parameters $\alpha$ and $\beta$.
\end{abstract}

Keywords. cosmology: miscellaneous, cosmology: observations, cosmology: theory

\section{Introduction}

The current observational data are still not able to decide between many different models proposed to explain the recent accelerated expansion of the universe. These models include, among others, modified gravitational theories and the addition of an exotic cosmological fluid, in the context of general relativity. As an alternative study, we can make a pure kinematical description of this recent phase of the universe, avoiding the choice of a gravitational theory and matter content. In this description one still models the universe as a metric manifold and uses the cosmological principle restricting the metric to the Friedmann-Lemaitre-Robertson-Walker (FLRW) metric. In this case, the deceleration function is given by

$$
q(z)=\frac{(1+z)}{H(z)} \frac{\mathrm{d} H(z)}{\mathrm{d} z}-1
$$

where $z$ is the redshift and $H(z)$ is the Hubble function.

Different approaches have been used in the literature in order to reconstruct some kinematic quantities such as the luminosity distance $D_{L}(z), H(z), q(z)$ and the jerk 

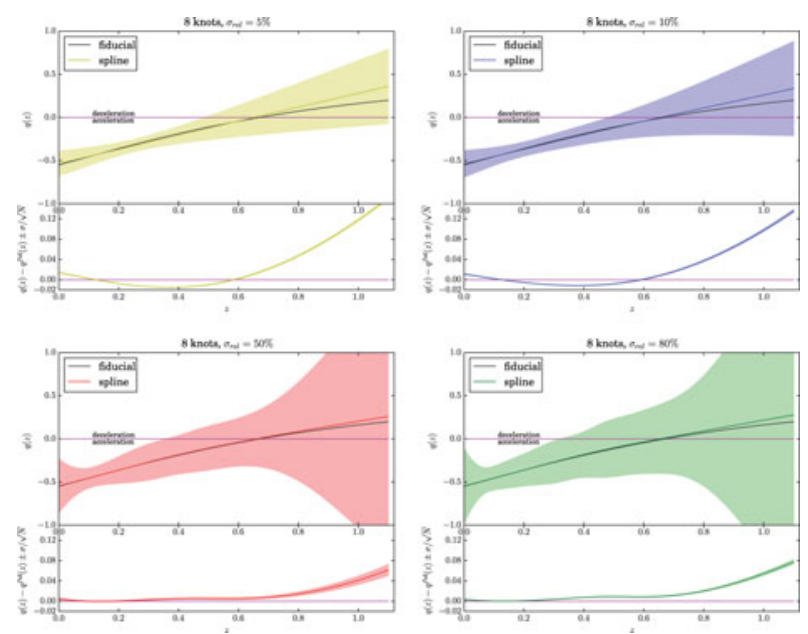

Figure 1. The top part of each panel shows the reconstructed curve of $q(z)$ (spline), computed using the mean values of the estimators, and its $1 \sigma$ error bar. Each panel displays the result obtained for a given $\sigma_{r e l}$. The bottom part shows the bias, $q(z)-q^{f i d}(z)$, and the $1 \sigma$ error bar of the mean curve, i.e., $\sigma / \sqrt{N}$.

function. One of these methods assumes a priori a functional form of the scale factor $a(t)\left(1+z=a_{0} / a\right)$, or equivalently $D_{L}(z), H(z)$ and so on (Riess et al. 2004, Visser 2004, Rapetti et al. 2007, Lu et al. 2011, Shafieloo 2012). This parametric reconstruction is strongly model dependent, so, besides having small variances the results present large biases.

In order to minimize the assumptions on the fitted curve, a second approach is to approximate the kinematic quantity by a piecewise nth-order polynomial function. This "non-parametric" reconstruction is dominated by the over-fitting feature, but it provides small biased estimators (see Daly et al. 2008 and Lazkoz et al. 2012).

\section{Methodology}

In this work, we describe $q(z)$ by a piecewise cubic polynomial function, also known as cubic spline. Imposing continuous second derivatives, the only free parameters of $q(z)$ are its values at the knots, i.e., $q\left(z_{i}\right)=q_{i}$, where $i$ runs from 0 to $n-1$ (being $n$ the number of knots). We then address both parametric and non-parametric methods including a penalty factor $P_{i}(\sigma)$ in the likelihood $L$, namely,

$$
F=-2 \ln (L)+\sum_{i=2}^{n-1} P_{i}(\sigma)
$$

where the penalty factor is given by

$$
P_{i}(\sigma)=\left(\left(\bar{q}_{i}-q_{i}\right) /\left(\sigma_{a b s}+\bar{q}_{i} \sigma_{r e l}\right)\right)^{2},
$$

$\bar{q}_{i}=\left(q_{i-1}+q_{i+1}\right) / 2$ and $\sigma_{a b s}=10^{-5}$. Varying the value of $\sigma_{r e l}$, see Figs. 1 and 2 , we are able to recover a full $n$ knots spline (over-fitting dominated), for large $\sigma_{r e l}$, and a single linear function in the entire redshift interval, for small $\sigma_{r e l}$, where $q_{i}$ 's are better constrained but they can be biased (if the assumed functional form significantly differs from the true one). 

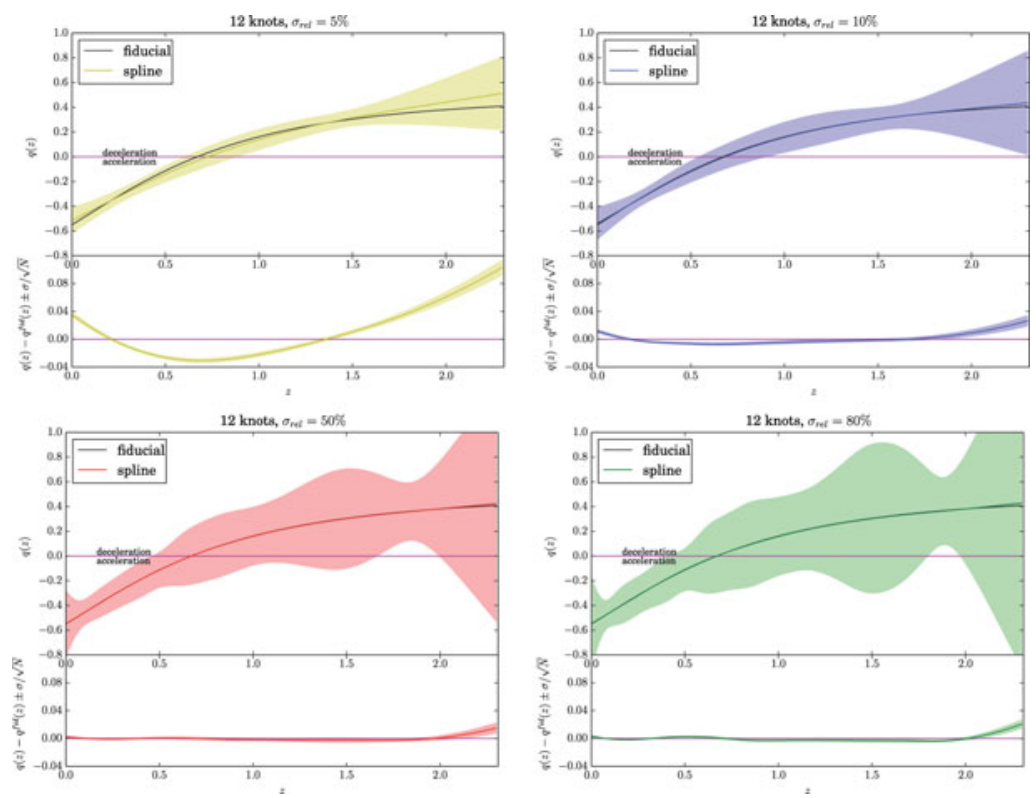

Figure 2. Equivalent to the caption of Fig. 1. These results were obtained considering Eq. 3.1 and 12 knots.

We use the SNeIa sample from SNLS3 (Conley et al. 2011 and Sullivan et al. 2011) and, thus, the likelihood is build as

$$
-2 \ln \left(L_{S N I a}\right)=\Delta \vec{m}^{T} C_{S N I a}^{-1} \Delta \vec{m}+\ln \left(\operatorname{det}\left(C_{S N I a}\right)\right),
$$

where $\mathrm{C}_{S N I a}=\mathrm{C}_{\text {stat }}(\alpha, \beta)+\mathrm{C}_{\text {sys }}$ is the data covariance and

$$
\Delta m_{i}=5 \log _{10}\left(D_{L}\left(z_{i}^{c m b}, z_{i}^{h e l}\right)\right)-\alpha\left(s_{i}-1\right)+\beta \mathcal{C}_{i}+M_{h_{i}}+5 \log _{10}\left(c / H_{0}\right)+25-m_{B i} .
$$

The SNIa phenomenological model contains four parameters $\alpha, \beta, M_{1}$ and $M_{2}$, where the first two are related to the stretch-luminosity and colour-luminosity, respectively, and $M_{1}$ and $M_{2}$ are absolute magnitudes.

We use data from Baryon Acoustic Oscillation (BAO) surveys such as WiggleZ, SDSS and 6dFGRS as described in Hinshaw et al. (2013). The BAO likelihood is given by

$$
-2 \ln \left(L_{B A O}\right)=\Delta \vec{d}_{v}^{T} C_{B A O}^{-1} \Delta \vec{d}_{v}
$$

where $C_{B A O}$ is the $\mathrm{BAO}$ constant covariance matrix and

$$
\Delta d_{v i}=d_{v i}-\frac{r_{r e c}}{D_{v}\left(z_{i}\right)} \text {. }
$$

The BAO depends on the comoving sound horizon at recombination $r_{r e c}$ and the geometric estimate of the effective distance $D_{v}\left(z_{i}\right)$. The last is calculated directly rewriting the distance as an integral of $q(z)$, however, $r_{r e c}$ have to be treated as an additional parameter since our model does not define $r_{r e c}$.

The Hubble function likelihood is build as

$$
-2 \ln \left(L_{H u b b l e}\right)=\Delta \vec{H}^{T} \mathrm{C}_{H u b b l e}^{-1} \Delta \vec{H},
$$

where $\mathrm{C}_{H \text { ubble }}$ is the diagonal constant covariance matrix and $\Delta H_{i}=H_{i}-H\left(z_{i}\right)$. This likelihood includes the $H(z)$ measurements which were obtained independently of BAO. 
The data is summarize, for example, in Zheng et al. (2014). We also include the $H_{0}$ $(z=0)$ measurement from Riess et al. (2011).

We apply the Monte Carlo (MC) approach to obtain the estimators of $q_{i}$ 's, $\alpha, \beta, M_{1}$ and $M_{2}$. For this, we first define a fiducial model from which $N$ random realizations will be generated. In particular, we assume a flat universe $(k=0)$ and a $\Lambda C D M$ cosmological model with $H_{0}=73 \mathrm{~km} \mathrm{~s}^{-1} \mathrm{Mpc}^{-1}, \Omega_{m}=0.3$ and $\Omega_{\Lambda}=0.7$. For each realization, the best-fitting values of the parameters are obtained by minimizing Eq. (2.1). At each step, i.e., for each resample, the arithmetic mean and the variance of the estimators are computed. This loop ends when the required precision is achieved. In this work, the number of realizations needed varies between $10^{4}$ and $2 \times 10^{5}$.

\section{Results and Concluding Remarks}

We first apply the methodology considering a spline of $q(z)$ with 8 knots within the redshift interval $[0.01,1.4]$. We perform this analysis using only the SNLS3 data, i.e., its covariance matrix to generate the realizations, and Eqs. (2.5) and (2.1) to obtain the means and variances of the $9 q_{i}$ estimators, $\alpha, \beta, M_{1}$ and $M_{2}$. We consider 4 different values of $\sigma_{r e l}=0.05,0.1,0.5$ and 0.8 . In Fig. 1 we show the reconstructed $q(z)$ function, computed using the mean of the estimators, and its $1 \sigma$ error bar.

In this second case we consider 12 knots within the redshift interval $[0,2.3]$. We perform this analysis using the joint likelihood:

$$
-2 \ln (L)=-2 \ln \left(L_{S N I a}\right)-2 \ln \left(L_{B A O}\right)-2 \ln \left(L_{\text {Hubble }}\right) .
$$

Fig. 2 displays the results for those 4 different $\sigma_{\text {rel }}$ values.

It is worth emphasizing that the parameters $\alpha, \beta, M_{1}, M_{2}$ and $H_{0}$ are recovered in all cases, i.e. ML estimators for these quantities have negligible biases.

In both figures we plotted the threshold line, $q(z)=0$, which indicates the deceleration/acceleration regions. In the 8 cases studied, we obtain the indication of an accelerated expansion with more than $1 \sigma$ confidence level in the interval $0 \leqslant z \lesssim 0.4$.

In this work, we used $\Lambda C D M$ as the fiducial model, in which $q(z)$ is almost linear. Therefore, in order to obtain a more conservative reconstruction of $q(z)$, it is necessary to apply this study for fiducial models with different functional forms of $q(z)$. Once we get suitable number of knots and $\sigma_{r e l}$, which work well for all fiducial models, we can apply the method using real data and recover a conservative estimate of the recent expansion history of the universe (Vitenti \& Penna-Lima, in prep.).

\section{References}

Conley, A., Guy, J., Sullivan, M., Regnault, N., \& Astier, P., et al. 2011, ApJS, 192, 1

Daly, R. A., Djorgovski, S. G., Freeman, K. A., Mory, M. P., \& O’Dea, C., et al. 2008, ApJ, 677,1

Hinshaw, G., Larson, D., Komatsu, E., \& Spergel, D. N. et al. 2013, ApJS, 208, 19

Lazkoz, R., Salzano, V., \& Sendra, I. 2012, Eur. Phys. J. C, 72, 2130

Lu, J., Xu, L., \& Liu, M. 2011, Phys. Lett. B, 699, 246

Rapetti, D., Allen, S. W., Amin, M. A., Blandford, R. D. 2007, MNRAS, 375, 1510

Riess, A. G., Strolger, L-G., Tonry, J., Casertano, S., Ferguson, H. C., et al. 2004, ApJ, 607, 665

Riess, A. G., Macri, L., Casertano, S., Lampeitl, H., Ferguson, H. C., et al. 2011, ApJ, 730, 119

Shafieloo, A. 2012, JCAP, 8, 2

Sullivan, M., Guy, J., Conley, A., Regnault, N., Astier, P., et al. 2011, ApJ, 737, 102

Turner, M. S. \& Riess, A. 2002, ApJ, 569, 18

Visser, M. 2004, Classical Quant. Grav., 21, 2603

Zheng, W., Li, H., Xia, J. Q. et al. 2014, Int. J. Mod. Phys. D, 23, 50051 